\title{
Kerncurriculum für das Studium des Schulfaches Pädagogik im Bachelor/Bakkalaureus- und Master/Magister-System
}

Erziehungswissenschaft 17 (2006) 32, S. 18-24

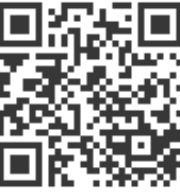

Quellenangabe/ Reference:

Kerncurriculum für das Studium des Schulfaches Pädagogik im Bachelor/Bakkalaureus- und Master/Magister-System - In: Erziehungswissenschaft 17 (2006) 32, S. 18-24 - URN:

urn:nbn:de:0111-opus-10674 - DOI: 10.25656/01:1067

https://nbn-resolving.org/urn:nbn:de:0111-opus-10674

https://doi.org/10.25656/01:1067

in Kooperation mit / in cooperation with:

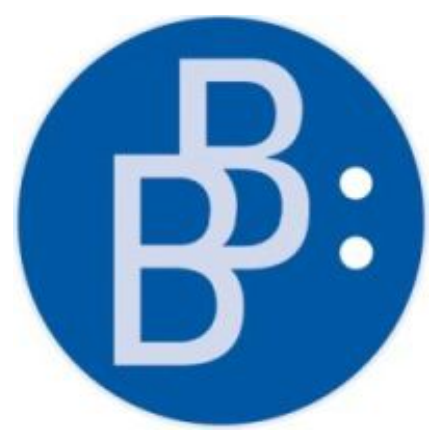

https://www.budrich.de

\section{Nutzungsbedingungen}

Gewährt wird ein nicht exklusives, nicht übertragbares, persönliches und beschränktes Recht auf Nutzung dieses Dokuments. Dieses Dokument ist ausschließlich für den persönlichen, nicht-kommerziellen Gebrauch bestimmt. Die Nutzung stellt keine Übertragung des Eigentumsrechts an diesem Dokument dar und gilt vorbehaltlich der folgenden Einschränkungen: Auf sämtlichen Kopien dieses Dokuments müssen alle Urheberrechtshinweise und sonstigen Hinweise auf gesetzlichen Schutz beibehalten werden. Sie dürfen dieses Dokument nicht in irgendeiner Weise abändern, noch dürfen Sie dieses Dokument für öffentliche oder kommerzielle Zwecke vervielfältigen, öffentlich ausstellen, aufführen, vertreiben oder anderweitig nutzen

Mit der Verwendung dieses Dokuments erkennen Sie die Nutzungsbedingungen an.

\section{Terms of use}

We grant a non-exclusive, non-transferable, individual and limited right to using this document.

This document is solely intended for your personal, non-commercial use. Use of this document does not include any transfer of property rights and it is conditional to the following limitations: All of the copies of this documents must retain all copyright information and other information regarding legal protection. You are not allowed to alter this document in any way, to copy it for public or commercial purposes, to exhibit the document in public, to perform, distribute or otherwise use the document in public.

By using this particular document, you accept the above-stated conditions of use.

\section{Kontakt / Contact:}

\section{peDOCS}

DIPF | Leibniz-Institut für Bildungsforschung und Bildungsinformation Informationszentrum (IZ) Bildung

E-Mail: pedocs@dipf.de

Internet: www.pedocs.de

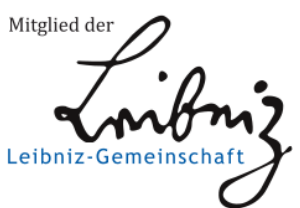




\section{INHALTSVERZEICHNIS}

Editorial

\section{Beiträge}

DGfE, Der Vorstand

Personelle Mindestausstattung im Fach Erziehungswissenschaft

DGfE, Der Vorstand

Kerncurriculum für das Studium des Schulfaches Pädagogik

im Bachelor/Bakkalaureus- und Master/Magister-System

DGfE, Der Vorstand

Strukturmodell für die Lehrerbildung im Bachelor/Bakkalaureusund Master/Magister-System

DGfE, Der Vorstand

Anonymisierung von Daten in der qualitativen Forschung:

Probleme und Empfehlungen

EWFT, 8. Plenarversammlung, 18.11.2005

Zur Problematik neuer Curricularnormwerte für die

Erziehungswissenschaft

EWFT, 8. Plenarversammlung, 18.11.2005

Strukturnotwendigkeiten für die Erziehungswissenschaft in

konsekutiven Hauptfachstudiengängen - Empfehlungen

Sieglinde Jornitz (DIPF)

Online Publizieren - auch in der Erziehungswissenschaft

\section{Mitteilungen des Vorstandes}

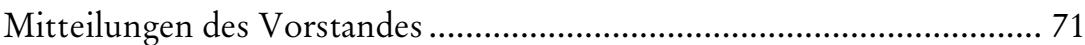

Kurzbericht aus der Akkreditierungsagentur ACQUIN ……………...... 77

Bericht von der UNESCO-Arbeit............................................................... 79

Networks on Didactics (EERA) ……………………………………...... 82 


\section{Berichte aus den Sektionen}

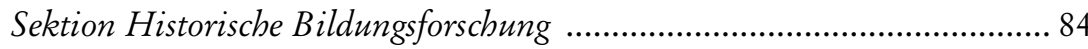

Sektion Allgemeine Erziehungswissenschaft

Kommission Bildungs- und Erziehungsphilosophie................................... 88

Kommission Pädagogische Anthropologie................................................. 91

Kommission Wissenschaftsforschung .................................................... 93

Kommission Erziehungswissenschaftliche Biographieforschung............ 94

Sektion International und Interkulturell Vergleichende

Erziebungswissenschaft

Kommission Vergleichende und interkulturelle

Erziehungswissenschaft 100

Sektion Empirische Bildungsforschung

Kommission Arbeitsgruppe für Empirische Pädagogische

Forschung (AEPF)

Kommission Bildungsorganisation, Bildungsplanung,

Bildungsrecht (KBBB)

Sektion Schulpädagogik

Kommission Professionsforschung und Lehrerbildung........................... 106

Kommission Grundschulforschung und Pädagogik der Primarstufe..... 108

Sektion Sonderpädagogik ........................................................................ 109

Sektion Berufs- und Wirtschaftspädagogik .............................................. 111

Sektion Sozialpädagogik

Kommission „Pädagogik der Frühen Kindheit“ ".................................... 112

Sektion Erwachsenenbildung ................................................................... 113

Sektion Freizeitforschung und Sportpädagogik

Kommission Pädagogische Freizeitforschung ....................................... 116

Sektion Franen- und Geschlechterforschung ............................................ 118

Sektion Medien- und Umweltpädagogik

Kommission Medienpädagogik

Kommission Bildung für eine nachhaltige Entwicklung........................ 129 
Sektion Differenzielle Erziehungs- und Bildungsforschung

Kommission „Pädagogik und Humanistische Psychologie“.

Kommission „Psychoanalytische Pädagogik“.....

\section{Notizen}

Notizen aus der Forschung

Notizen aus der Wissenschafts- und Bildungspolitik

Ausschreibungen/Preise....

Tagungskalender.

Personalia... 175 
DGfE, Der Vorstand, 10. Dezember 2005

\section{Kerncurriculum für das Studium des Schulfaches Pädagogik im Bachelor/Bakkalaureus- und Master/Magister-System}

\section{Präambel}

Pädagogik ist in zahlreichen allgemeinbildenden Schulen (Realschulen, gymnasialen Oberstufen) und beruflichen Schulen (Berufsfachschulen) in einer Reihe von Bundesländern Teil des Fächerangebots. Die universitäre Ausbildung der Lehrkräfte für dieses Schulfach ist bis heute unbefriedigend geblieben. Aus diesem Grund hat sich der Vorstand der DGfE entschieden, ein Kerncurriculum für das Studium des Schulfaches Pädagogik an allgemeinbildenden und beruflichen Schulen vorzulegen. Die Empfehlung des Kerncurriculums gilt für alle Schularten und Schulstufen; auf seiner Grundlage sollte die Ausgestaltung der Studienmodule nach den jeweiligen schulart- und schulstufenspezifischen Anforderungen erfolgen. Der Vorstand ergänzt damit seine Empfehlungen »Kerncurriculum für das Hauptfachstudium Erziehungswissenschaft « vom 31. Januar 2004 und "Strukturmodell für die Lehrerbildung im Bachelor-Master-System « vom 11. Dezember 2004 in der Fassung vom 10. Dezember 2005. Aus den beiden Empfehlungen ergeben sich die Inhalte und strukturellen Rahmenvorgaben für das Studium des Schulfaches Pädagogik. Das Kerncurriculum des Hauptfachstudiums Erziehungswissenschaft umfasst neben den Praktika vier Studieneinheiten:

(1) Grundlagen der Erziehungswissenschaft;

(2) gesellschaftliche, politische und rechtliche Bedingungen von Bildung, Ausbildung und Erziehung in schulischen und nicht-schulischen Handlungsfeldern unter Einschluss internationaler Aspekte;

(3) Bildungsforschung und forschungsmethodische Grundlagen; und

(4) Einführung in erziehungswissenschaftliche Studienrichtungen. 
In Anlehnung hieran werden für das Schulfach Pädagogik sieben Studieneinheiten vorgesehen, die sich auf das Bachelor- und das Master-Studium erstrecken.

\section{Strukturbeschreibung}

Aus dem Strukturmodell für die Lehrerbildung im Bachelor-MasterSystem ergeben sich für das Bachelor-Studium des Schulfaches Pädagogik

(1) fachwissenschaftliche Studien im Umfang von 60 LP (ca. 40 SWS) und

(2) berufswissenschaftliche Studien im Gesamtumfang von 53 LP (ca. 34 SWS). Das Studium der Berufswissenschaft umfasst einerseits ein berufsorientierendes Angebot in Verbindung mit einem Industrie- oder Dienstleistungspraktikum im Umfang von 12 LP (ca. 8 SWS) sowie andererseits erziehungswissenschaftliche, pädagogisch-psychologische, bildungssoziologische und fachdidaktische Studienanteile im Umfang von 26 LP (ca. 16 SWS) und ein begleitetes Schulpraktikum im Umfang von 15 LP (ca. 10 SWS).

Der Master-Studiengang des Schulfaches Pädagogik umfasst (1) die schulformbezogene Fortsetzung des Schulfaches Pädagogik im Umfang von $15 \mathrm{LP}$ (ca. 10 SWS), (2) fachdidaktische Studien im Umfang von $9 \mathrm{LP}$ (ca. 6 SWS) mit einem fachdidaktischen Praktikum im Umfang von 7,5 LP (ca. 5 SWS), d.h. in einem Gesamtumfang von 16,5 LP, und (3) berufswissenschaftliche Studien im Umfang von 42 LP (ca. 28 SWS).

\section{Empfehlung zum Bachelor/Bakkalaures-Studiengang}

Da Inhalte der Studieneinheiten 1-3 bereits in den erziehungswissenschaftlichen Anteilen der Berufswissenschaft enthalten sind, ist die Ausgestaltung der Module für das Schulfach Pädagogik auf eine Erweiterung und Vertiefung der erziehungswissenschaftlichen Kenntnisse und Kompetenzen gerichtet; eine Überschneidung mit den erziehungswissenschaftlichen Anteilen der Berufswissenschaft ist auszuschließen. 


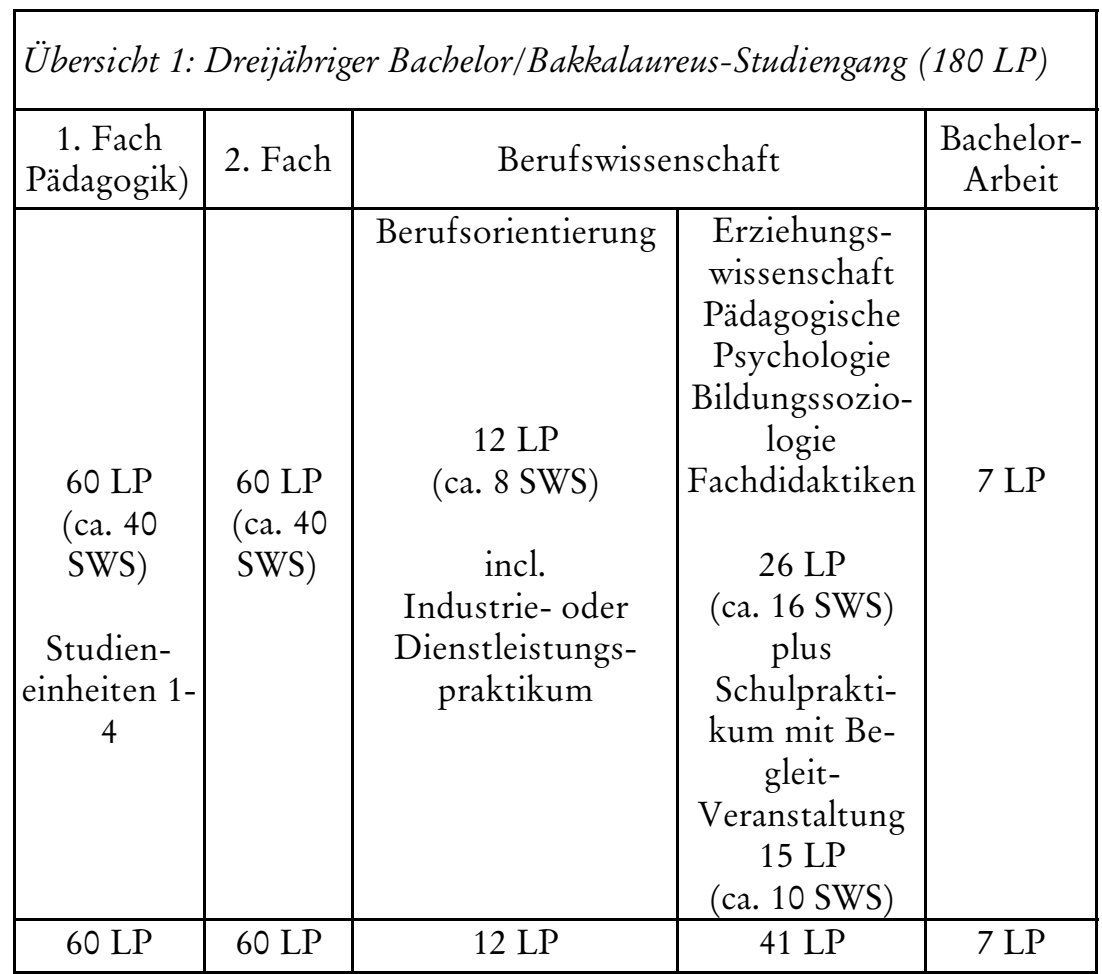

\section{Studieneinheit 1: Grundlagen der Erziehungswissenschaft}

Aufgaben: Unterscheidung zwischen lebensweltlichen pädagogischen Vorstellungen und erziehungswissenschaftlichen Denkweisen; Befähigung, pädagogische Probleme begrifflich einzugrenzen, in ihrem geschichtlichen Kontext zu verstehen und theoretische Ansätze zu differenzieren.

\begin{tabular}{|c|l|}
\hline 1.1 & $\begin{array}{l}\text { Grundbegriffe der Erziehungswissenschaft und ihrer Teildiszi- } \\
\text { plinen (insbesondere Erziehung, Sozialisation, Lehren und } \\
\text { Lernen, Unterricht, Bildung, Ausbildung, Generation, Ent- } \\
\text { wicklung und Lebenslauf) }\end{array}$ \\
\hline 1.2 & Geschichte und Theorie der Erziehung und Bildung \\
\hline 1.3 & Wissenschaftstheoretische Ansätze der Erziehungswissenschaft \\
\hline
\end{tabular}


Studieneinheit 2: Gesellschaftliche, politische und rechtliche Bedingungen von Bildung, Ausbildung und Erziehung in schulischen und nichtschulischen Einrichtungen unter Einschluss internationaler Aspekte

Aufgaben: Einführung in empirische und sozialhistorische Bedingungen pädagogischen Handelns und erziehungswissenschaftlicher Fragestellungen in nationaler und internationaler Perspektive; Befähigung zur kritischen Auseinandersetzung mit erziehungswissenschaftlicher Forschung und pädagogischen Aufgabenstellungen im Hinblick auf ihre historischen, kulturellen, politischen und rechtlichen Rahmenbedingungen; Befähigung zur Beurteilung und Entwicklung von Handlungskonzepten.

2.1 Theorien, Funktionen und geschichtliche Aspekte von Bildungs-, Erziehungs- und Hilfeinstitutionen sowie von Sozialisationsinstanzen

2.2 Bildungspolitik, Bildungsrecht und Bildungsorganisation sowie Systeme der sozialen Sicherung unter Einschluss international vergleichender Fragestellungen

2.3 Differenz und Gleichheit, kulturelle und soziale Heterogenität

Studieneinheit 3: Einführung in erziehungswissenschaftliche Studienrichtungen

Aufgaben: Verknüpfung des grundlegenden erziehungswissenschaftlichen Wissens und Könnens mit einzelnen Studienrichtungen; Überblick über die Gliederung der Gesamtdisziplin; Einführung in die Theorie und Geschichte von Teildisziplinen und Berufsfeldern.

3.1 Struktur der Erziehungswissenschaft in der Pluralität ihrer Teildisziplinen

3.2 Handlungsfelder, Handlungsformen, Einstellungen und Haltungen im Kontext einer Studienrichtung 


\begin{tabular}{|c|c|}
\hline \multicolumn{2}{|c|}{ Studieneinheit 4: Fachdidaktik I } \\
\hline \multicolumn{2}{|r|}{$\begin{array}{l}\text { Aufgaben: Einführung in die Problematik der Auswahl und Anord- } \\
\text { nung von Lehr-Lerneinheiten und Themen des Fachunterrichts; Be- } \\
\text { fähigung zur Beurteilung administrativer Lehrplanvorgaben auf der } \\
\text { Grundlage von Kenntnissen zur Fachgeschichte und zu didaktischen } \\
\text { Alternativen; Fähigkeit zur Nutzung der Freiräume in Richtlinien vor } \\
\text { dem Hintergrund von Kenntnissen zu didaktischen Theorien }\end{array}$} \\
\hline 4.1 & Lehrplanentwicklung des Pädagogikunterrichts \\
\hline 4.2 & Einführung in fachdidaktische Theorieansätze \\
\hline 4.3 & Vertiefung eines didaktischen Theorieansatzes \\
\hline
\end{tabular}

\section{Empfehlung zum Master/Magister-Studiengang}

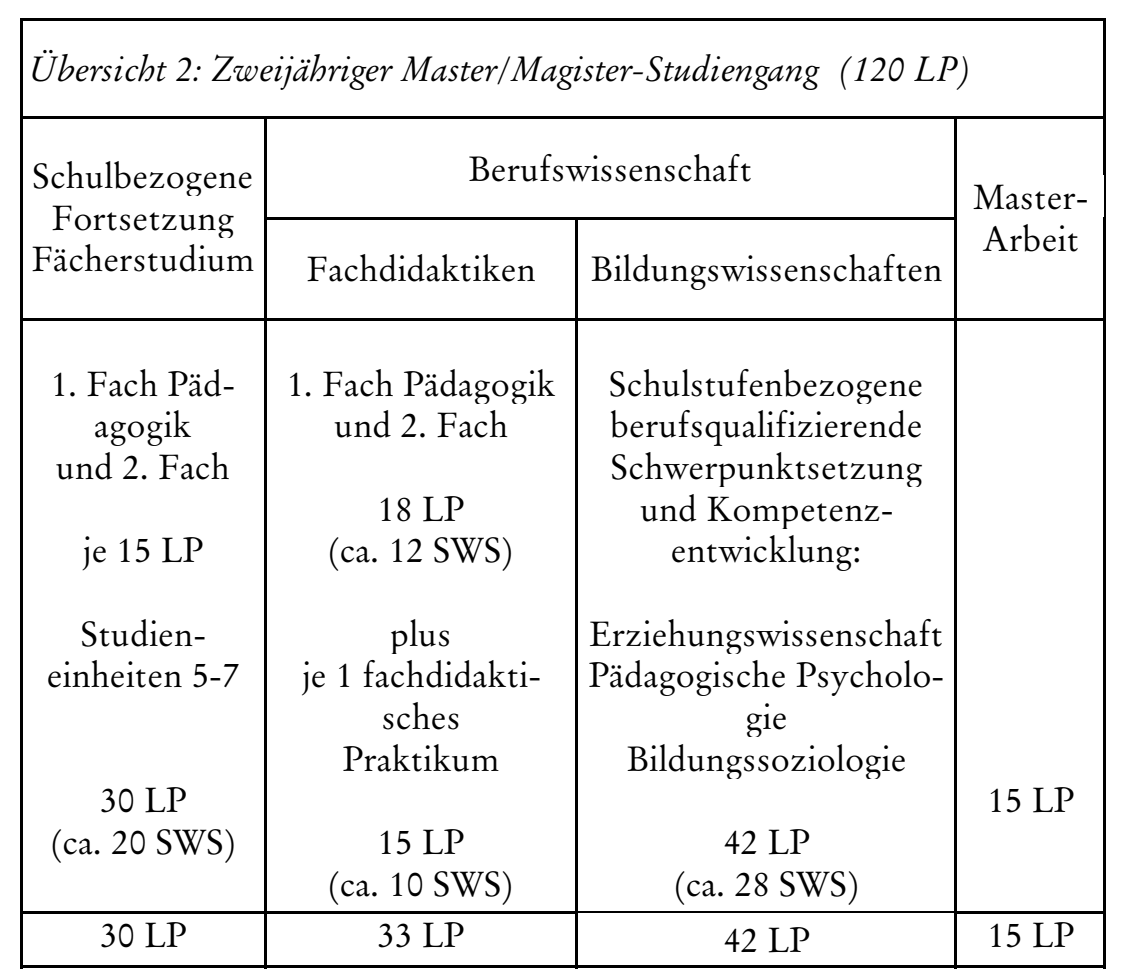


Die Studieneinheiten des Master-Studiums vertiefen das fachwissenschaftliche, das fachdidaktische und das berufswissenschaftliche Lehrerbildungsstudium (Studieneinheiten 5-7). Auf diese Studieneinheiten sind 33 LP (Fachstudium Pädagogik: 15 LP; Fachdidaktik: 18 LP) zu verteilen. Die Studieneinheit 7 ist nicht in Untereinheiten strukturiert, um unterschiedliche Varianten der Verbindung fachdidaktischer Themen und Fragestellungen mit fachwissenschaftlichen und berufswissenschaftlichen Aspekten zu ermöglichen.

Bei der Modularisierung der Studieneinheiten des MA-Studiums im Schulfach Pädagogik ist eine Überschneidung mit den erziehungswissenschaftlichen Anteilen der Bildungswissenschaften auszuschließen.

Studieneinheit 5: Bildungsforschung und forschungsmethodische Grundlagen

Aufgaben: Einführung in die Bildungsforschung; Einführung in erziehungswissenschaftliche Forschungsmethoden; Befähigung zu grundlegenden forschungsmethodischen Unterscheidungen; Einübung in den Gebrauch mindestens eines Forschungsverfahrens

\begin{tabular}{|c|l|}
\hline 5.1 & Qualitative und quantitative Methoden \\
\hline 5.2 & $\begin{array}{l}\text { Erziehungswissenschaftlich besonders relevante Ansätze (z.B. } \\
\text { pädagogisches Fallverstehen, Hermeneutik, Phänomenologie, } \\
\text { Diagnostik, Evaluation, Bildungsstatistik) }\end{array}$ \\
\hline 5.3 & $\begin{array}{l}\text { Erziehungs- und Bildungsforschung (z.B. Benachteiligtenfor- } \\
\text { schung, Lehr-Lernforschung, Weiterbildungsforschung) }\end{array}$ \\
\hline
\end{tabular}




\section{Studieneinheit 6: Fachdidaktik II}

Aufgaben: Vertiefende Auseinandersetzung mit planerischen und fachmethodischen Aspekten des Pädagogikunterrichts; Verdeutlichung der Schwierigkeiten bei der Verbindung von Wissenschaftspropädeutik und Handlungspropädeutik; Befähigung zur Erkenntnis der interdisziplinären Dimensionen des Faches und deren unterrichtsmethodischer Verknüpfung im Zusammenhang mit dem Projektansatz.

Die Studieneinheit ist mit einem fachdidaktischen Praktikum im Mindestumfang von 7 bis 8 LP verbunden.

\begin{tabular}{|l|l|}
\hline 6.1 & Unterrichtsinhalte und -methoden des Pädagogikunterrichts \\
\hline 6.2 & Textanalyse und Praxisanalyse \\
\hline 6.3 & Projekte im Pädagogikunterricht \\
\hline
\end{tabular}

\section{Studieneinheit 7: Fachdidaktisches Lehrforschungsprojekt}

Aufgaben: Im fachdidaktischen Lehrforschungsprojekt verfolgen die Studierenden unter Anleitung aus der Forschungsperspektive eine fachdidaktische Forschungsfrage im Kontext von Beobachtungen und Übungen im schulischen Pädagogikunterricht. In Verbindung mit den Studieneinheiten 5 und 6 werden in der Studieneinheit 7 fachdidaktische, fachwissenschaftliche und berufswissenschaftliche Perspektiven miteinander verknüpft.

In diese Studieneinheit ist ein fachdidaktisches Praktikum im Mindestumfang von 9 LP integriert.

Abkürzungen:

BA Bachelor/Bakkalaures

MA Master/Magister

LP Leistungspunkte

SWS Semesterwochenstunden 